\title{
Emotional discrimination during viewing unpleasant pictures: timing in human anterior ventrolateral prefrontal cortex and amygdala
}

\author{
Satoru Kohno ${ }^{1}{ }^{*}$, Madoka Noriuchi $^{1}$, Yoshinobu Iguchi ${ }^{1}$, Yoshiaki Kikuchi ${ }^{2}$ and Yoko Hoshi ${ }^{1}$ \\ Tokyo Metropolitan Institute of Medical Science, Tokyo, Japan \\ ${ }^{2}$ Graduate School of Tokyo Metropolitan University, Tokyo, Japan
}

\section{Edited by:}

Hans-Jochen Heinze, University of Magdeburg, Germany

Reviewed by:

Annette Beatrix Bruehl, University of Cambridge, UK

Mattie Tops, VU University

Amsterdam, Netherlands

${ }^{*}$ Correspondence:

Satoru Kohno, Tokyo Metropolitan

Institute of Medical Science, 2-1-6

Kamikitazawa, Setagaya-ku Tokyo

156-8506, Japan

e-mail: kohno-st@igakuken.or.jp
The ventrolateral prefrontal cortex (VLPFC) and amygdala have critical roles in the generation and regulation of unpleasant emotions, and in this study the dynamic neural basis of unpleasant emotion processing was elucidated by using paired-samples permutation $t$-tests to identify the timing of emotional discrimination in various brain regions. We recorded the temporal dynamics of blood-oxygen-level-dependent (BOLD) signals in those brain regions during the viewing of unpleasant pictures by using functional magnetic resonance imaging (fMRI) with high temporal resolution, and we compared the time course of the signal within the volume of interest (VOI) across emotional conditions. Results show that emotional discrimination in the right amygdala precedes that in the left amygdala and that emotional discrimination in both those regions precedes that in the right anterior VLPFC. They support the hypotheses that the right amygdala is part of a rapid emotional stimulus detection system and the left amygdala is specialized for sustained stimulus evaluation and that the right anterior VLPFC is implicated in the integration of viscerosensory information with affective signals between the bilateral anterior VLPFCs and the bilateral amygdalae.

Keywords: functional magnetic resonance imaging (fMRI), IAPS pictures, amygdala, emotion, emotional discrimination, ventrolateral prefrontal cortex, timing estimation

\section{INTRODUCTION}

The human brain contains neural circuits responsible for the emotional responses elicited by stimuli, and the amygdala and prefrontal cortex (PFC) are key components of these circuits (Davidson and Irwin, 1999). The bilateral ventrolateral prefrontal cortices (VLPFCs) are involved, through the amygdalae, in both the generation and regulation of unpleasant emotions (Wager et al., 2008; Hoshi et al., 2011). Furthermore, recent functional magnetic resonance imaging (fMRI) studies have shown functional connectivity between the VLPFC and the amygdala (Banks et al., 2007; Guyer et al., 2008; Tang et al., 2013; Townsend et al., 2013). For instance, when subjects reappraise unpleasant scenes in order to decrease the strength of the unpleasant feelings elicited, VLPFC activity is inversely correlated with amygdala activity (Ochsner et al., 2002; Wager et al., 2008). In contrast, attenuated VLPFC function and/or heightened amygdala activation has been found in manic bipolar patients and high-anxiety adolescents when they were compared with healthy subjects (Foland et al., 2008; Guyer et al., 2008). Although fMRI research on functional connectivity has clarified temporal correlations between the activity an unpleasant picture stimulus elicits in the VLPFC and the activity it elicits in the amygdala, the dynamic neural basis of unpleasant emotion processing in these regions remains unclear.
Recently, our parametric mediation analysis of the activity affective pictures elicit in the VLPFCs, amygdalae, visual cortex, premotor cortex, supplementary motor area, fusiform gyrus and dorsolateral PFC found the activities in these regions to be negatively related with the valence ratings of affective pictures and found that the right anterior VLPFC (BA47) was only a mediator of the valence ratings, while the other brain regions were both sources and mediators (Kida et al., 2012). It has been widely accepted that the anterior VLPFC receives several kinds of sensory input (olfactory, gustatory, visceral, somatic, and visual) as well as limbic inputs from the amygdala, entorhinal and perirhinal cortex, and subiculum (Price, 1999). The right anterior VLPFC has therefore been hypothesized to be implicated in the integration of viscerosensory information with affective signals (Lévesque et al., 2004). It has also been hypothesized that the right amygdala is part of a rapid or dynamic system for detecting emotional stimuli and the left amygdala is specialized for sustained stimulus evaluation (Wright et al., 2001). If the right VLPFC plays a role in integrating emotional information, emotional discrimination in the right anterior VLPFC would occur after that in other brain regions. If the right amygdala were part of a rapid emotional stimulus detection system, the emotional discrimination there would precede that in the left amygdala. Here, emotional discrimination means regional brain reacts to unpleasant stimuli differently 
from neutral stimuli according to the study by Sabatinelli et al. (2009).

In the present study we examined the timing of emotional discrimination in the bilateral VLPFCs and the bilateral amygdalae in order to test the above-mentioned hypotheses about the roles of these brain regions in unpleasant emotion processing. We did this by focusing on three key brain regionsthe visual cortex, amygdala and VLPFC - and using fMRI with high temporal resolution: $300 \mathrm{~ms}$. Though the blood-oxygenlevel-dependent (BOLD) signal in $\mathrm{FMRI}$ is delayed relative to the neural response, the timing of the signal within active regions is highly reliable (Kim et al., 1997; Menon and Kim, 1999; Miezin et al., 2000). Comparing the time course of the signal within the volume of interest (VOI) across emotional conditions eliminated potential confounding factors regarding the individual differences in BOLD signal timing (Aguirre et al., 1998; Buxton et al., 1998) and variations in vascular anatomy (Sabatinelli et al., 2009). Furthermore, the time course in the primary visual cortex was also investigated in order to verify the validity of our results.

\section{MATERIALS AND METHODS SUBJECTS}

Nineteen right-handed healthy volunteers (13 male, 6 female, mean age $20.4 \pm 1.8$ years) participated in the present study. None had a history of neurological or psychiatric disorders, and written informed consent was obtained from all of them. The ethics committee of the Tokyo Metropolitan Institute of Medical Science approved the study.

\section{STIMULI AND PROCEDURE}

To select appropriate stimuli, we had the emotional valence of 137 pictures from the International Affective Picture System (IAPS; Lang et al., 2008) and 100 pictures from other photograph collections (Fuji Film Co., Tokyo, Japan) rated by 33 healthy volunteers (12 male, 21 female, 20-28 years old), none of which was a subject in the present study (Hoshi et al., 2011). The emotional valence was rated on a digital scale with nine grades ranging from 1 (very unpleasant) to 9 (very pleasant).

Ninety pictures for which the subjects' ratings coincided were selected as stimuli: 30 unpleasant pictures (IAPS image numbers 3170, 1275, 9561, 7380, 9400, 3500, 1111, 1300, 9313, 2800, 8230, 9570, 9008, 9410, 7360, 3301, 3350, 9300, 3400, $9405,1280,2095,9290,1090,3160,6313,9440,3180,6560$ and 9007), 30 neutral pictures (IAPS image numbers 7025,
$7041,7090,7100,7140,7115,7211,7009,7705,7004,7150$, $7235,6150,7006,7503,7224,7233,7950,7010,2221,7034$, 2520, 7000 and 5500) and 30 pleasant pictures (IAPS image numbers 1710, 1027, 1040, 2091, 2023, 1440, 7325, 2025 and 8005) (Hoshi et al., 2011). The "rating coincided" means that 33 volunteers rated a picture at the same valence range (1-3, 4-6, 7-9). The selected pictures included content such as mutilation, threatening animals, angry people, pollution, accidents, grief, nature, pets, children, neutral objects and neutral people. Erotic pictures were excluded because sex differences in the emotions they evoke have been seen (Sabatinelli et al., 2004). Experimental runs consisted of 30 picture presentations, and bias was avoided by randomly presenting 10 unpleasant, 10 neutral and 10 pleasant during each run. Each picture was presented for $3.0 \mathrm{~s}$ and was followed by a 9.0-s presentation of a white crosshair in the middle of the screen (Figure 1). As the purpose of our study was to investigate emotional discrimination during viewing unpleasant pictures, only unpleasant and neutral pictures (controls) might have been sufficient. However, to reduce psychological and neural effects of anticipation, we adopted a multiple randomization method, in which pleasant pictures were also presented in addition to unpleasant and neutral pictures. Under the experimental conditions, it is difficult for subjects to predict which kind of stimuli will be presented. Furthermore, in the present study, differences in BOLD signals between unpleasant and neutral stimuli were examined, which is expected to effectively reduce anticipation-related brain activations. Our fMRI experiment consisted of three runs, each using a different set of pictures. After the runs the subjects rated the emotional valence of each of the 90 pictures, as experienced at the time of the initial presentation, on a scale ranging from 1 (very unpleasant) to 9 (very pleasant) using SelfAssessment Manikin (SAM) rating methods (Bradley and Lang, 1994).

\section{ACOUISITION}

The picture stimuli were delivered via a goggle system (Resonance Technology, Northridge, CA). The subjects were instructed to look at the crosshair in the middle of the screen, remain still and not fall asleep. The experiments were performed using a Philips Achieva 3.0 T MRI scanner using gradient echo planar imaging, and 1,230 volumes per run were obtained from four oblique axial slices including the bilateral amygdalae, the bilateral anterior VLPFCs (BA47) and the primary visual cortex (V1). The slice position

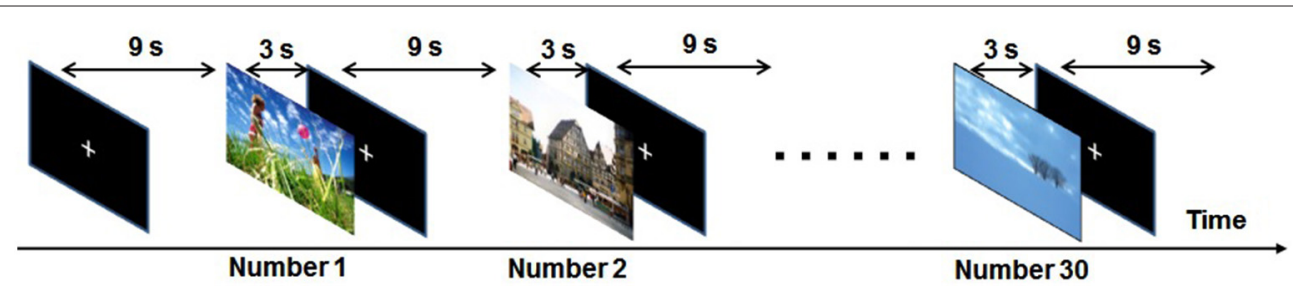

FIGURE 1 | Schematic diagram of the experimental paradigm in one run. During each run 30 picture stimuli (10 unpleasant, 10 neutral and 10 pleasant) were randomly presented for $3.0 \mathrm{~s}$ and each was followed by a 9.0-s white cross-hair in the middle of the screen. 


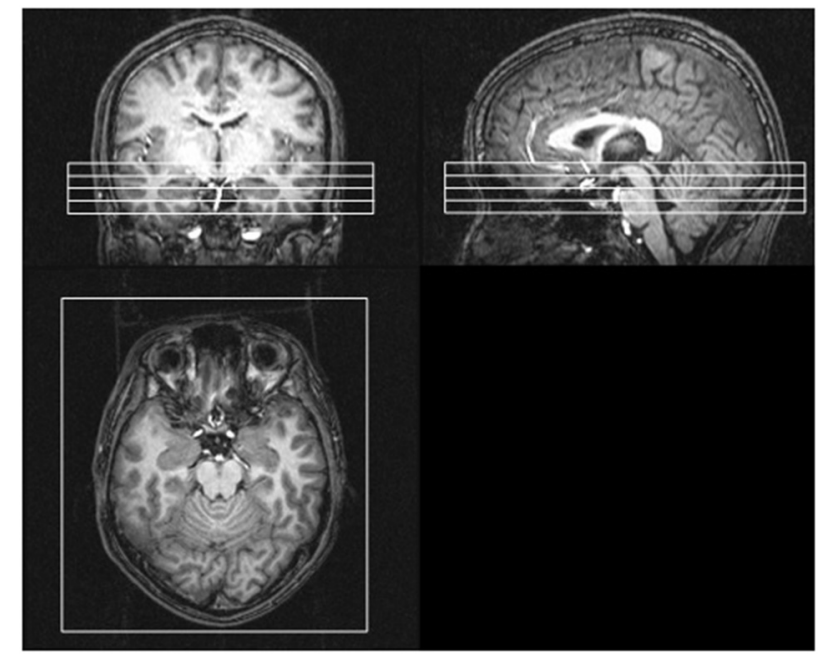

FIGURE 2 | Positions of four oblique axial slices involving the bilateral amygdalae, the bilateral anterior VLPFCs (BA47) and the primary visual cortex. The slice position was decided by aligning the top slice with the anterior commissure and the posterior commissure (AC-PC) line.

was decided by aligning the top slice with the anterior commissure and the posterior commissure (AC-PC) line (Figure 2).

The acquisition parameters were as follows: repetition time $=300 \mathrm{~ms}$, echo time $=30 \mathrm{~ms}$, flip angle $=40^{\circ}$, field of view $=230 \mathrm{~mm}$, matrix $=128 \times 80$, number of slices $=4$, slice thickness $=5 \mathrm{~mm}$, slice gap $=1 \mathrm{~mm}$, interleaved acquisition. The flip angle parameter was adjusted to maximize the MRI signals from gray matter under the condition of the fast repetition time (300 ms). This run was repeated three times. In addition, highresolution whole-brain anatomical images were acquired using three-dimensional T1-weighted sequence (repetition time $=7.8$ $\mathrm{ms}$, echo time $=3.8 \mathrm{~ms}$, inversion time $=975.8 \mathrm{~ms}$, field of view $=$ $240 \mathrm{~mm}$, matrix $=256 \times 240$, number of slices $=160$, slice volume $=60 \mathrm{~mm}$, slice thickness $=1 \mathrm{~mm}$ ).

\section{DATA PROCESSING}

We identified the activation maps of the fMRI data by using SPM8 software. ${ }^{1}$ To detrend and analyze the fMRI timecourse data, we used a multiple linear-regression algorithm based on the general linear model and then used spatial smoothing with a Gaussian filter (full width at half maximum $=8 \mathrm{~mm}$ ). All the MRI data were analyzed with a canonical hemodynamic response function (Friston et al., 1998) identical to the activation time course, and an appropriate threshold value (uncorrected, $p<0.05$ ) was used for the generation of $t$ maps. In each subject, the centers of the VOIs were set to the coordinates for peak $t$ values in the bilateral anterior VLPFCs, the bilateral amygdalae and the primary visual cortex responding to both unpleasant and neutral picture stimuli. BOLD signals were averaged over a VOI with a $5-\mathrm{mm}$ radius (Figure 3), and the average value in each brain region was

${ }^{1}$ www.fil.ion.ucl.ac.uk/spm/
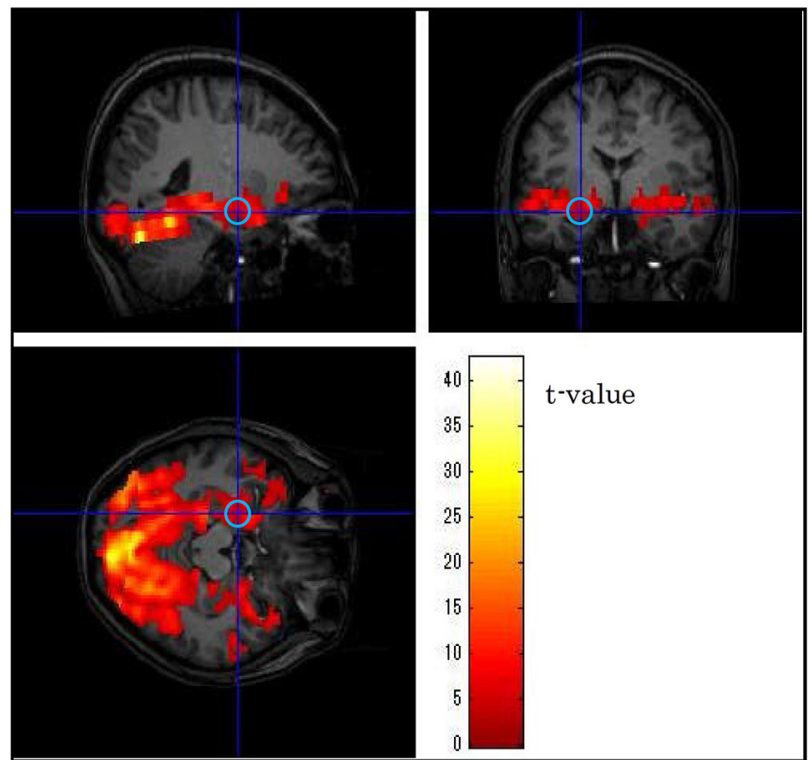

FIGURE 3 | Example of the position of a volume of interest (left amygdala) in one subject. The central position was determined using as guides neuroanatomical atlases and three-dimensional activation maps.

extracted from the fMRI data by using the MarsBar tool for SPM. $^{2}$ Although our objective was to investigate the timing of emotional discrimination in the bilateral VLPFCs and the bilateral amygdalae, the BOLD signal in the primary visual cortex was also extracted in order to verify the validity of the results.

The BOLD signals for each run were linearly detrended. The baseline levels for the signals were set by the averaging in the $3.0 \mathrm{~s}$ period ( 10 points) just prior to the first stimulation epoch in order to acquire baseline signals after steady-state magnetism, and the percent signal changes were calculated. Then the signals were folded into a single epoch by averaging the unpleasant stimulus epochs and the neutral stimulus epochs in each run. Here we confirmed that the differences of the signal changes between unpleasant and neutral picture stimuli at stimulus onset were very small (less than $0.1 \%$ ). Thus the folded plots were set to zero at the stimulus onset in order to compare the response time courses of the BOLD signals activated by unpleasant and neutral picture stimuli (Kohno et al., 2009).

\section{STATISTICAL ANALYSIS}

To reliably identify the timing (defined in this paper as the time after stimulus onset) of the BOLD signals of emotional discrimination between unpleasant and neutral picture stimuli in the bilateral amygdalae, the bilateral anterior VLPFCs and the primary visual cortex, paired-samples permutation $t$-tests (a straightforward way to solve the multiple-comparisons problem) were performed for each time point in the folded plots (Figure 4; Blair and Karniski, 1993; Maris and Oostenveld, 2007). The $p$ values at each time point were calculated from

\footnotetext{
${ }^{2}$ http://marsbar.sourcefoge.net/
} 


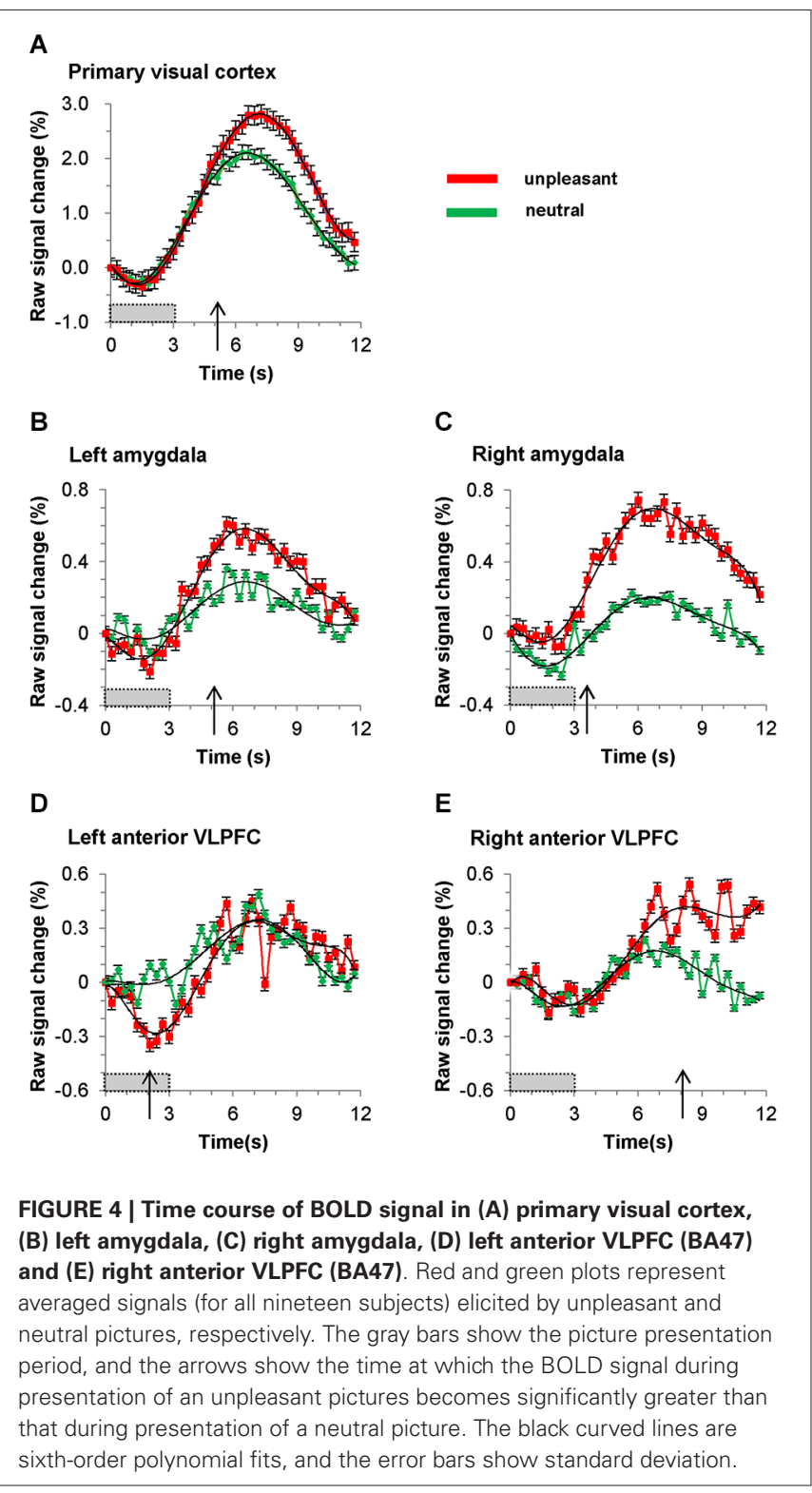

the permutation distribution (number of permutations $=2^{19}$ $=524,288)$. The time of emotional discrimination was defined as the first time after stimulus onset at which the $p$ value was less than five percent for more than two successive points. To find the data trends, negative peak and positive peak, we used the least-squares method to fit a sixth-order polynomial to the sets of measured data (see black curves in Figure 4).

\section{RESULTS \\ VALENCE RESULTS}

The valence ratings of our sample had a mean of $3.08(S D=0.77)$ for unpleasant pictures, $5.07(S D=0.24)$ for neutral pictures and $6.58(S D=0.71)$ for pleasant pictures. The valence rating results in the present study differed significantly between unpleasant and neutral pictures $\left(p<10^{-12}\right.$; two-sample $t$-test).

\section{fMRI RESULTS}

The folded time courses for the unpleasant and the neutral picture stimuli, averaged over all subjects, are shown in Figure 4 . The timing of emotional discrimination during negative emotional stimuli is listed in Table 1. Also listed there are the times and percent signal changes of both positive and negative peaks (times and changes calculated from sixth-order polynomial fittings). In our data both the negative peaks and the positive peaks were found in all time courses of BOLD signals in the brain regions. During unpleasant picture presentations the BOLD signal at the positive peak of the response was greater, relative to that at the positive peak during neutral picture presentations, in the primary visual cortex $(p<0.001$; paired $t$-test), the left amygdala ( $p<0.05$; paired $t$-test), the right amygdala ( $p<0.001$; paired $t$-test) and the right anterior VLPFC ( $p<0.0001$; paired $t$-test). And during unpleasant picture presentations the BOLD signal at the negative peak of the response was greater, relative to that during neutral picture presentations, in the left anterior VLPFC ( $p<0.01$; paired $t$-test). The time courses of positive peaks activated by unpleasant picture stimuli were steeper, relative to those of positive peaks activated by neutral picture stimuli, in the bilateral amygdalae and the left anterior VLPFC, whereas in the primary visual cortex and the right anterior VLPFC those time courses were almost the same.

The response for BOLD signals in the primary visual cortex during unpleasant and neutral stimuli showed transient decreases just after stimulus onset. The response dipped after the onset of the visual stimulus, reaching a minimum value of $-0.28 \%$ at $1.2 \mathrm{~s}$, at which time the response began to recover, and became positive at $2.4 \mathrm{~s}$. Within $4.5 \mathrm{~s}$ of the onset of a stimulus there was almost no difference (less than $0.1 \%$ ) between unpleasant and neutral picture stimuli with regard to the BOLD signals in the primary visual cortex, while the BOLD signals in the bilateral amygdalae and the left anterior VLPFC clearly differed between unpleasant and neutral picture stimuli. After $4.5 \mathrm{~s}$ the time course of the BOLD signals in the primary visual cortex differed between unpleasant and neutral picture stimuli.

The timing of emotional discrimination during unpleasant picture stimuli (indicated by $p<0.05$ for more than two successive points; paired permutation $t$-test) was $2.1 \mathrm{~s}$ in the left anterior VLPFC, $3.6 \mathrm{~s}$ in the right amygdala, $5.1 \mathrm{~s}$ in the left amygdala, $5.1 \mathrm{~s}$ in the primary visual cortex and $8.1 \mathrm{~s}$ in the right anterior VLPFC.

\section{DISCUSSION}

Investigating the timing of emotional discrimination in the bilateral VLPFCs and the bilateral amygdalae during the viewing of unpleasant pictures, we found that the emotional discrimination in the right amygdala preceded that in the left amygdala and that the emotional discrimination in both these regions preceded that in the right anterior VLPFC.

\section{PRIMARY VISUAL CORTEX}

The results about the initial BOLD-signal dip in the primary visual cortex were quite consistent with those of previous studies (Duong et al., 2000; Kim et al., 2000; Behzadi and Liu, 2006; 
Table 1 | Timing of emotional discrimination in bilateral VLPFCs, bilateral amygdalae and the primary visual cortex.

\begin{tabular}{llcc}
\hline Region & Stimulus & $\begin{array}{c}\text { Negative peak time (s)/ } \\
\text { signal changes (\%) }\end{array}$ & $\begin{array}{c}\text { Positive peak time (s)/ } \\
\text { signal changes (\%) }\end{array}$ \\
\hline Primary visual cortex & Unpleasant & $1.3 /-0.30$ & $\begin{array}{c}\text { Timing of emotional } \\
\text { discrimination (s) }\end{array}$ \\
& Neutral & $1.1 /-0.28$ & $7.1 / 2.8$ \\
Left amygdala & Unpleasant & $1.6 /-0.15$ & $6.5 / 2.1$ \\
& Neutral & $1.8 /-0.03$ & $6.5 / 0.58$ \\
Right amygdala & Unpleasant & $1.4 /-0.04$ & $6.6 / 0.29$ \\
& Neutral & $1.7 /-0.18$ & $6.7 / 0.7$ \\
Left anterior VLPFC & Unpleasant & $2.4 /-0.28$ & $6.5 / 0.20$ \\
& Neutral & $1.8 /-0.01$ & $7.1 / 0.34$ \\
Right anterior VLPFC & Unpleasant & $2.9 /-0.12$ & $7.2 / .35$ \\
& Neutral & $2.5 /-0.13$ & $8.3 / 0.42$ \\
\end{tabular}

$\mathrm{Hu}$ and Yacoub, 2012). In addition, the consistency of the early time course ( $<4.5 \mathrm{~s}$ after stimulus onset) between unpleasant and neutral picture stimuli means that the primary visual cortex is not directly involved in the initial emotional discrimination. These results seem to be reasonable.

The late time course $(>4.5 \mathrm{~s})$ of V1 BOLD signals differed between unpleasant picture stimuli and neutral picture stimuli. The projections from the amygdala to the primary visual cortex were confirmed using retrograde tracers in macaque monkeys and chimpanzees (Freese and Amaral, 2005, 2006), and the visual emotion discrimination has been thought to result from reentrant feedback from the amygdala to the visual cortex (Iwai and Yukie, 1987; LeDoux, 1998; Herrmann et al., 2008). The BOLD-signal difference between unpleasant and neutral stimuli after $4.5 \mathrm{~s}$ is accounted for by the reentrant feedback from the amygdala to the visual cortex because emotional discrimination in the right amygdala preceded that in the primary visual cortex by $\sim 1.5 \mathrm{~s}$.

\section{AMYGDALA}

A recent meta-analysis of functional imaging studies (Sergerie et al., 2008), unlike some previous studies, did not find evidence for amygdala lateralization in terms of sex or valence but instead provided strong support for a functional dissociation between left and right amygdala in terms of temporal dynamics (Phillips et al., 2001; Wright et al., 2001). In our experiment the emotional discrimination in the right amygdala preceded that in the left amygdala by $\sim 1.5 \mathrm{~s}$. Indeed it has been hypothesized that the right amygdala is part of a rapid or dynamic emotional stimulus detection system and the left amygdala might be specialized for sustained stimulus evaluations (Wright et al., 2001), and our results about the timing of emotional discrimination in the human amygdala support that hypothesis.

\section{ANTERIOR VLPFC}

The left anterior VLPFC (BA47) is responsible for controlled retrieval of semantic information (Wagner et al., 2001; Gold and Buckner, 2002; Velanova et al., 2003; Badre and Wagner, 2007). It should be noted that the subjects in our experiment had to interpret the meaning of the IAPS pictures because the pictures contain various complicated scenes. After that, the meanings of pictures provided to the subjects would be appraised in order to transform a percept into something that elicits emotion (Smith and Ellsworth, 1985). Thus our result that the left anterior VLPFC was implicated in the earliest emotional discrimination (2.1 s) is reasonable because the semantic processing of unpleasant pictures is likely to be a higher perceptual load than the semantic processing of neutral pictures (Eastwood et al., 2001).

The right VLPFC has been characterized as important for response inhibition (Aron et al., 2004; Aron and Poldrack, 2005; Lieberman et al., 2007; Hampshire et al., 2010; Levy and Wagner, 2011; Cohen et al., 2013). A recent meta-analysis has revealed that both middle and posterior subregions of the right VLPFC (i.e., BA45 and BA44) are consistently active during the Go/No-Go and Stop-Signal tasks used to measure the response inhibition, whereas no evidence for the involvement of the right anterior VLPFC (BA47) has been found (Levy and Wagner, 2011). However, it has been hypothesized that the right anterior VLPFC is implicated in the integration of viscerosensory information with affective signals (Lévesque et al., 2004). The integration is supposed to be the last event in the processing of the viscerosensory information. Therefore our result that the emotional discrimination in the right anterior VLPFC occurs after that in the other brain regions investigated in this study supports the above-mentioned hypothesis. Interestingly, unlike the BOLD signals in the other brain regions, those in the right anterior VLPFC remained high after the emotional discrimination of unpleasant picture stimuli. It has been reported that subjective emotional experience involves a relatively long time and that feeling occurs over several tens of seconds (Lutz et al., 2002; Mauss et al., 2005). Alternatively, it is also conceivable that the prolonged right anterior VLPFC activation is due to anticipation (Waugh et al., 2008; Tops et al., 2014). This anticipatory activation would typically be triggered by signs that an aversive stimulus may be imminent. The zigzag pattern in the right anterior VLPFC after the finished emotional stimulus might represent physiological phenomenon. At the moment, there is no valid explanation for the zigzag pattern, however, it should be focused on the future work. Thus the time courses of BOLD signals and the late timing of emotional discrimination in the right anterior VLPFC during the viewing of unpleasant pictures might reflect the mental experience and/or anxious anticipation. 
In conclusion, we determined differences in the timing of emotional discrimination in the bilateral anterior VLPFCs and the bilateral amygdalae during the viewing of unpleasant pictures. Emotional discrimination in those brain regions occurred last in the right anterior VLPFC. The present findings imply that the right anterior VLPFC integrates viscerosensory information. They also indicate that the right amygdala is part of a rapid emotional stimulus detection system and the left amygdala is specialized for sustained stimulus evaluation.

\section{REFERENCES}

Aguirre, G. K., Zarahn, E., and D'esposito, M. (1998). The variability of human, BOLD hemodynamic responses. Neuroimage 8, 360-369. doi: 10.1006/nimg. 1998.0369

Aron, A. R., and Poldrack, R. A. (2005). The cognitive neuroscience of response inhibition: relevance for genetic research in attention-deficit/hyperactivity disorder. Biol. Psychiatry 57, 1285-1292. doi: 10.1016/j.biopsych.2004.10.026

Aron, A. R., Robbins, T. W., and Poldrack, R. A. (2004). Inhibition and the right inferior frontal cortex. Trends Cogn. Sci. 8, 170-177. doi: 10.1016/j.tics.2004.02. 010

Badre, D., and Wagner, A. D. (2007). Left ventrolateral prefrontal cortex and the cognitive control of memory. Neuropsychologia 45, 2883-2901. doi: 10.1016/j. neuropsychologia.2007.06.015

Banks, S. J., Eddy, K. T., Angstadt, M., Nathan, P. J., and Phan, K. L. (2007). Amygdala-frontal connectivity during emotion regulation. Soc. Cogn. Affect. Neurosci. 2, 303-312. doi: 10.1093/scan/nsm029

Behzadi, Y., and Liu, T. T. (2006). Caffeine reduces the initial dip in the visual BOLD response at 3 T. Neuroimage 32, 9-15. doi: 10.1016/j.neuroimage.2006.03.005

Blair, R. C., and Karniski, W. (1993). An alternative method for significance testing of waveform difference potentials. Psychophysiology 30, 518-524. doi: 10.1111/j. 1469-8986.1993.tb02075.x

Bradley, M. M., and Lang, P. J. (1994). Measuring emotion: the self-assessment manikin and the semantic differential. J. Behav. Ther. Exp. Psychiatry 25, 49-59. doi: 10.1016/0005-7916(94)90063-9

Buxton, R. B., Wong, E. C., and Frank, L. R. (1998). Dynamics of blood flow and oxygenation changes during brain activation: the balloon model. Magn. Reson. Med. 39, 855-864. doi: 10.1002/mrm.1910390602

Cohen, J. R., Berkman, E. T., and Lieberman, M. D. (2013). "Intentional and incidental self-control in ventrolateral PFC," in Principles of Frontal Lobe Function, 2nd Edn. eds D. T. Stuss and R. T. Knight (New York: Oxford University Press), 417-440.

Davidson, R. J., and Irwin, W. (1999). The functional neuroanatomy of emotion and affective style. Trends Cogn. Sci. 3, 11-21. doi: 10.1016/s13646613(98)01265-0

Duong, T. Q., Kim, D. S., Uğurbil, K., and Kim, S. G. (2000). Spatiotemporal dynamics of the BOLD fMRI signals: toward mapping submillimeter cortical columns using the early negative response. Magn. Reson. Med. 44, 231-242. doi: 10.1002/1522-2594(200008)44:2<231::aid-mrm10>3.3.co;2-k

Eastwood, J. D., Smilek, D., and Merikle, P. M. (2001). Differential attentional guidance by unattended faces expressing positive and negative emotion. Percept. Psychophys. 63, 1004-1013. doi: 10.3758/bf03194519

Foland, L. C., Altshuler, L. L., Bookheimer, S. Y., Eisenberger, N., Townsend, J., and Thompson, P. M. (2008). Evidence for deficient modulation of amygdala response by prefrontal cortex in bipolar mania. Psychiatry Res. 162, 27-37. doi: 10.1016/j.pscychresns.2007.04.007

Freese, J. L., and Amaral, D. G. (2005). The organization of projections from the amygdala to visual cortical areas TE and V1 in the macaque monkey. J. Comp. Neurol. 486, 295-317. doi: 10.1002/cne.20520

Freese, J. L., and Amaral, D. G. (2006). Synaptic organization of projections from the amygdala to visual cortical areas TE and V1 in the macaque monkey. J. Comp. Neurol. 496, 655-667. doi: 10.1002/cne.20945

Friston, K. J., Fletcher, P., Josephs, O., Holmes, A., Rugg, M. D., and Turner, R. (1998). Event-related fMRI: characterizing differential responses. Neuroimage 7, 30-40. doi: 10.1006/nimg.1997.0306

Gold, B. T., and Buckner, R. L. (2002). Common prefrontal regions coactivate with dissociable posterior regions during controlled semantic and phonological tasks. Neuron 35, 803-812. doi: 10.1016/s0896-6273(02)00800-0
Guyer, A. E., Lau, J. Y., McClure-Tone, E. B., Parrish, J., Shiffrin, N. D., Reynolds, R. C., et al. (2008). Amygdala and ventrolateral prefrontal cortex function during anticipated peer evaluation in pediatric social anxiety. Arch. Gen. Psychiatry 65, 1303-1312. doi: 10.1001/archpsyc.65.11.1303

Hampshire, A., Chamberlain, S. R., Monti, M. M., Duncan, J., and Owen, A. M. (2010). The role of the right inferior frontal gyrus: inhibition and attentional control. Neuroimage 50, 1313-1319. doi: 10.1016/j.neuroimage.2009.12.109

Herrmann, M. J., Huter, T., Plichta, M. M., Ehlis, A. C., Alpers, G. W., Mühlberger, A., et al. (2008). Enhancement of activity of the primary visual cortex during processing of emotional stimuli as measured with event-related functional nearinfrared spectroscopy and event-related potentials. Hum. Brain Mapp. 29, 2835. doi: 10.1002/hbm.20368

Hoshi, Y., Huang, J., Kohri, S., Iguchi, Y., Naya, M., Okamoto, T., et al. (2011). Recognition of human emotions from cerebral blood flow changes in the frontal region: a study with event-related near-infrared spectroscopy. J. Neuroimaging 21, e94-e101. doi: 10.1111/j.1552-6569.2009.00454.x

$\mathrm{Hu}, \mathrm{X}$., and Yacoub, E. (2012). The story of the initial dip in fMRI. Neuroimage 62, 1103-1108. doi: 10.1016/j.neuroimage.2012.03.005

Iwai, E., and Yukie, M. (1987). Amygdalofugal and amygdalopetal connections with modality-specific visual cortical areas in macaques (macaca fuscata, M. mulatta and M. fascicularis). J. Comp. Neurol. 261, 362-387. doi: 10.1002/cne.9026 10304

Kida, I., Iguchi, Y., and Hoshi, Y. (2012). "Convergence of emotion processing on the right ventrolateral prefrontal cortex: parametric mediation analysis of fMRI," in 20th Annual Meeting and Exhibition International Society of Magnetic Resonance in Medicine (Melbourne, Australia).

Kim, D. S., Duong, T. Q., and Kim, S. G. (2000). High-resolution mapping of iso-orientation columns by fMRI. Nat. Neurosci. 3, 164-169. doi: 10.1038/ 72109

Kim, S. G., Richter, W., and Uğurbil, K. (1997). Limitations of temporal resolution in functional MRI. Magn. Reson. Med. 37, 631-636. doi: 10.1002/mrm. 1910370427

Kohno, S., Sawamoto, N., Urayama, S., Aso, T., Aso, K., Seiyama, A., et al. (2009). Water-diffusion slowdown in the human visual cortex on visual stimulation precedes vascular responses. J. Cereb. Blood Flow Metab. 29, 1197-1207. doi: 10. 1016/j.neures.2009.09.634

Lang, P. J., Bradley, M. M., and Cuthbert, B. N. (2008). International Affective Picture System (IAPS): Affective Ratings of Pictures and Instruction Manual. Technical Report A-8. Gainesville, FL: University of Florida.

LeDoux, J. (1998). Fear and the brain: where have we been and where are we going? Biol. Psychiatry 44, 1229-1238. doi: 10.1016/s0006-3223(98)00282-0

Lévesque, J., Joanette, Y., Mensour, B., Beaudoin, G., Leroux, J. M., Bourgouin, P., et al. (2004). Neural basis of emotional self-regulation in childhood. Neuroscience 129, 361-369. doi: 10.1016/j.neuroscience.2004.07.032

Levy, B. J., and Wagner, A. D. (2011). Cognitive control and right ventrolateral prefrontal cortex: reflexive reorienting, motor inhibition and action updating. Ann. N Y Acad. Sci. 1224, 40-62. doi: 10.1111/j.1749-6632.2011. 05958.x

Lieberman, M. D., Eisenberger, N. I., Crockett, M. J., Tom, S. M., Pfeifer, J. H., and Way, B. M. (2007). Putting feelings into words: affect labeling disrupts amygdala activity in response to affective stimuli. Psychol. Sci. 18, 421-428. doi: 10.1111/j. 1467-9280.2007.01916.x

Lutz, A., Lachaux, J. P., Martinerie, J., and Varela, F. J. (2002). Guiding the study of brain dynamics by using first-person data: synchrony patterns correlate with ongoing conscious states during a simple visual task. Proc. Natl. Acad. Sci. U S A 99, 1586-1591. doi: 10.1073/pnas.032658199

Maris, E., and Oostenveld, R. (2007). Nonparametric statistical testing of EEG- and MEG-data. J. Neurosci. Methods 164, 177-190. doi: 10.1016/j.jneumeth.2007.03. 024

Mauss, I. B., Levenson, R. W., McCarter, L., Wilhelm, F. H., and Gross, J. J. (2005). The tie that binds? Coherence among emotion experience, behavior and physiology. Emotion 5, 175-190. doi: 10.1037/1528-3542.5.2.175

Menon, R. S., and Kim, S. G. (1999). Spatial and temporal limits in cognitive neuroimaging with fMRI. Trends Cogn. Sci. 3, 207-216. doi: 10.1016/s13646613(99)01329-7

Miezin, F. M., Maccotta, L., Ollinger, J. M., Petersen, S. E., and Buckner, R. L. (2000). Characterizing the hemodynamic response: effects of presentation rate, sampling procedure and the possibility of ordering brain activity based on relative timing. Neuroimage 11, 735-759. doi: 10.1006/nimg.2000.0568 
Ochsner, K. N., Bunge, S. A., Gross, J. J., and Gabrieli, J. D. (2002). Rethinking feelings: an FMRI study of the cognitive regulation of emotion. J. Cogn. Neurosci. 14, 1215-1229. doi: 10.1162/089892902760807212

Phillips, M. L., Medford, N., Young, A. W., Williams, L., Williams, S. C., Bullmore, E. T., et al. (2001). Time courses of left and right amygdalar responses to fearful facial expressions. Hum. Brain Mapp. 12, 193-202. doi: 10.1002/10970193(200104)12:4\%3C193::AID-HBM1015\%3E3.0.CO;2-A

Price, J. L. (1999). Prefrontal cortical networks related to visceral function and mood. Ann. N Y Acad. Sci. 877, 383-396. doi: 10.1111/j.1749-6632.1999. tb09278.x

Sabatinelli, D., Flaisch, T., Bradley, M. M., Fitzsimmons, J. R., and Lang, P. J. (2004). Affective picture perception: gender differences in visual cortex? Neuroreport 15, 1109-1112. doi: 10.1097/00001756-200405190-00005

Sabatinelli, D., Lang, P. J., Bradley, M. M., Costa, V. D., and Keil, A. (2009). The timing of emotional discrimination in human amygdala and ventral visual cortex. J. Neurosci. 29, 14864-14868. doi: 10.1523/jneurosci.327809.2009

Sergerie, K., Chochol, C., and Armony, J. L. (2008). The role of the amygdala in emotional processing: a quantitative meta-analysis of functional neuroimaging studies. Neurosci. Biobehav. Rev. 32, 811-830. doi: 10.1016/j.neubiorev.2007. 12.002

Smith, C. A., and Ellsworth, P. C. (1985). Patterns of cognitive appraisal in emotion. J. Pers. Soc. Psychol. 48, 813-838. doi: 10.1037//0022-3514.48.4.813

Tang, Y., Kong, L., Wu, F., Womer, F., Jiang, W., Cao, Y., et al. (2013). Decreased functional connectivity between the amygdala and the left ventral prefrontal cortex in treatment-naive patients with major depressive disorder: a restingstate functional magnetic resonance imaging study. Psychol. Med. 43, 19211927. doi: 10.1017/S0033291712002759

Tops, M., Boksem, M. A., Quirin, M., IJzerman, H., and Koole, S. L. (2014). Internally directed cognition and mindfulness: an integrative perspective derived from predictive and reactive control systems theory. Front. Psychol. 5:429. doi: 10.3389/fpsyg.2014.00429

Townsend, J. D., Torrisi, S. J., Lieberman, M. D., Sugar, C. A., Bookheimer, S. Y., and Altshuler, L. L. (2013). Frontal-amygdala connectivity alterations during emotion downregulation in bipolar I disorder. Biol. Psychiatry 73, 127-135. doi: 10.1016/j.biopsych.2012.06.030
Velanova, K., Jacoby, L. L., Wheeler, M. E., McAvoy, M. P., Petersen, S. E., and Buckner, R. L. (2003). Functional-anatomic correlates of sustained and transient processing components engaged during controlled retrieval. J. Neurosci. 23, 8460-8470.

Wager, T. D., Davidson, M. L., Hughes, B. L., Lindquist, M. A., and Ochsner, K. N. (2008). Prefrontal-subcortical pathways mediating successful emotion regulation. Neuron 59, 1037-1050. doi: 10.1016/j.neuron.2008.09.006

Wagner, A. D., Paré-Blagoev, E. J., Clark, J., and Poldrack, R. A. (2001). Recovering meaning: left prefrontal cortex guides controlled semantic retrieval. Neuron 31 , 329-338. doi: 10.1016/s0896-6273(01)00359-2

Waugh, C. E., Wager, T. D., Fredrickson, B. L., Noll, D. C., and Taylor, S. F. (2008). The neural correlates of trait resilience when anticipating and recovering from threat. Soc. Cogn. Affect. Neurosci. 3, 322-332. doi: 10.1093/scan/ nsn024

Wright, C. I., Fischer, H., Whalen, P. J., McInerney, S. C., Shin, L. M., and Rauch, S. L. (2001). Differential prefrontal cortex and amygdala habituation to repeatedly presented emotional stimuli. Neuroreport 12, 379-383. doi: 10 . 1097/00001756-200102120-00039

Conflict of Interest Statement: The authors declare that the research was conducted in the absence of any commercial or financial relationships that could be construed as a potential conflict of interest.

Received: 11 June 2014; accepted: 19 January 2015; published online: 10 February 2015

Citation: Kohno S, Noriuchi M, Iguchi Y, Kikuchi Y and Hoshi Y (2015) Emotional discrimination during viewing unpleasant pictures: timing in human anterior ventrolateral prefrontal cortex and amygdala. Front. Hum. Neurosci. 9:51. doi: 10.3389/fnhum.2015.00051

This article was submitted to the journal Frontiers in Human Neuroscience.

Copyright (C) 2015 Kohno, Noriuchi, Iguchi, Kikuchi and Hoshi. This is an open-access article distributed under the terms of the Creative Commons Attribution License (CC BY). The use, distribution and reproduction in other forums is permitted, provided the original author(s) or licensor are credited and that the original publication in this journal is cited, in accordance with accepted academic practice. No use, distribution or reproduction is permitted which does not comply with these terms. 